\title{
Nuclear Dependence of Transverse Single-Spin Asymmetries in Polarized $p+A$ Collisions at RHIC
}

\author{
Stephen Pate* [for the PHENIX Collaboration] \\ Physics Department, New Mexico State University, Las Cruces NM 88003, USA \\ E-mail: pate@nmsu.edu
}

\begin{abstract}
Large transverse single-spin asymmetries (TSSA) in hadron production at forward rapidity have been observed in polarized $p+p$ interactions for many decades, over a large range of center-ofmass energies, and have led to the investigation of spin-momentum correlations such as the Sivers and Collins effects. In the last few years, it has been discovered at RHIC that these single-spin asymmetries may be enhanced or suppressed in $p+A$ collisions, and the nuclear-size and centrality dependence have been studied. A variety of phenomena have been observed and likely they do not have all a single explanation; we see an apparent quenching of the TSSA in forward charged hadron production with increasing nuclear size, while we see an enhancement in the asymmetry in $J / \psi$ production, and in very forward neutron production even a sign change in the asymmetry is seen. Other systems, such as $\pi^{0}$ production at central rapidity, do not display a nuclear-size dependence. These observations provide a bridge between the study of the initial state in heavyion collisions and that of the nucleon spin puzzle, and open up a new method for the investigation of cold nuclear matter.
\end{abstract}

23rd International Spin Physics Symposium - SPIN2018 -

10-14 September, 2018

Ferrara, Italy

${ }^{*}$ Speaker. 


\section{Transverse Single Spin Asymmetries in $\vec{p}+p$ Interactions}

Large transverse single-spin asymmetries (TSSAs) have been observed in forward hadron production in $\vec{p}+p$ interactions for many decades and continue to be a subject of experimental and theoretical investigation. (And they are the topic of many talks at this conference!) A major result of the multi-decades study of this phenomenon is an understanding of the importance of various spin-momentum correlations.

- Initial state correlations: For example, the Sivers effect correlates the transverse spin of the nucleon with the intrinsic transverse momentum of the resident quark [14].

- Final state correlations: For example, the Collins effect correlates the transverse spin of the fragmenting quark with the transverse momentum of the produced hadron [8].

Direct measurements of these functions in semi-inclusive deep-inelastic scattering and in $e^{+} e^{-}$ annihilation interactions can be applied back to $\vec{p}+p$ interactions to reproduce data with some success [5]. We have learned that fragmentation (i.e. the Collins effect) seems to be the main culprit for large asymmetries see in $\vec{p}+p \rightarrow \pi+X$ [10], at least in the $x_{F}<0.3$ region; for $x_{F}>0.3$ other mechanisms must be invoked as well [4]. An excellent overview of this field can be found in the review article by Pitonyak [13].

The main focus of this presentation is: What happens when these interactions occur in a nuclear environment?

\section{Transverse Single Spin Asymmetries in $\vec{p}+A$ Interactions}

The nuclear environment brings many additional physics processes into play with many possible outcomes for these asymmetries. We have been aware since the 1980s of the changes the nucleus can have on the observed nucleon parton distribution functions (the EMC effect and nuclear shadowing), which represents a modification of the initial state. Gluon saturation and/or the existence of a Color Glass Condensate may also modify the magnitude of TSSAs. Multiple rescatterings of the final state particles may result in a broadening of the transverse distribution functions (leading to a dilution of the asymmetry) or to a modification of the effective $x_{F}$ (which might enhance the asymmetry). Ultra-peripheral collisions can induce very strong electromagnetic fields and Primakoff-like effects.

An important issue that arises in the analysis of $p+A$ interactions is the centrality of the interaction; how closely did the proton pass near the center of the nucleus? The impact parameter determines the average number of binary $p+p$ and $p+n$ collisions $\left(N_{\mathrm{coll}}^{\mathrm{Avg}}\right)$ that will occur as the proton passes through the nucleus. Of course the impact parameter cannot be measured directly; instead we observe the the charged-particle multiplicity at extreme rapidity in a set of "BeamBeam Counters" and use this to establish a "centrality classification" from $0 \%$ to $100 \%$. A model calculation then determines $N_{\text {coll }}^{\mathrm{Avg}}$. 


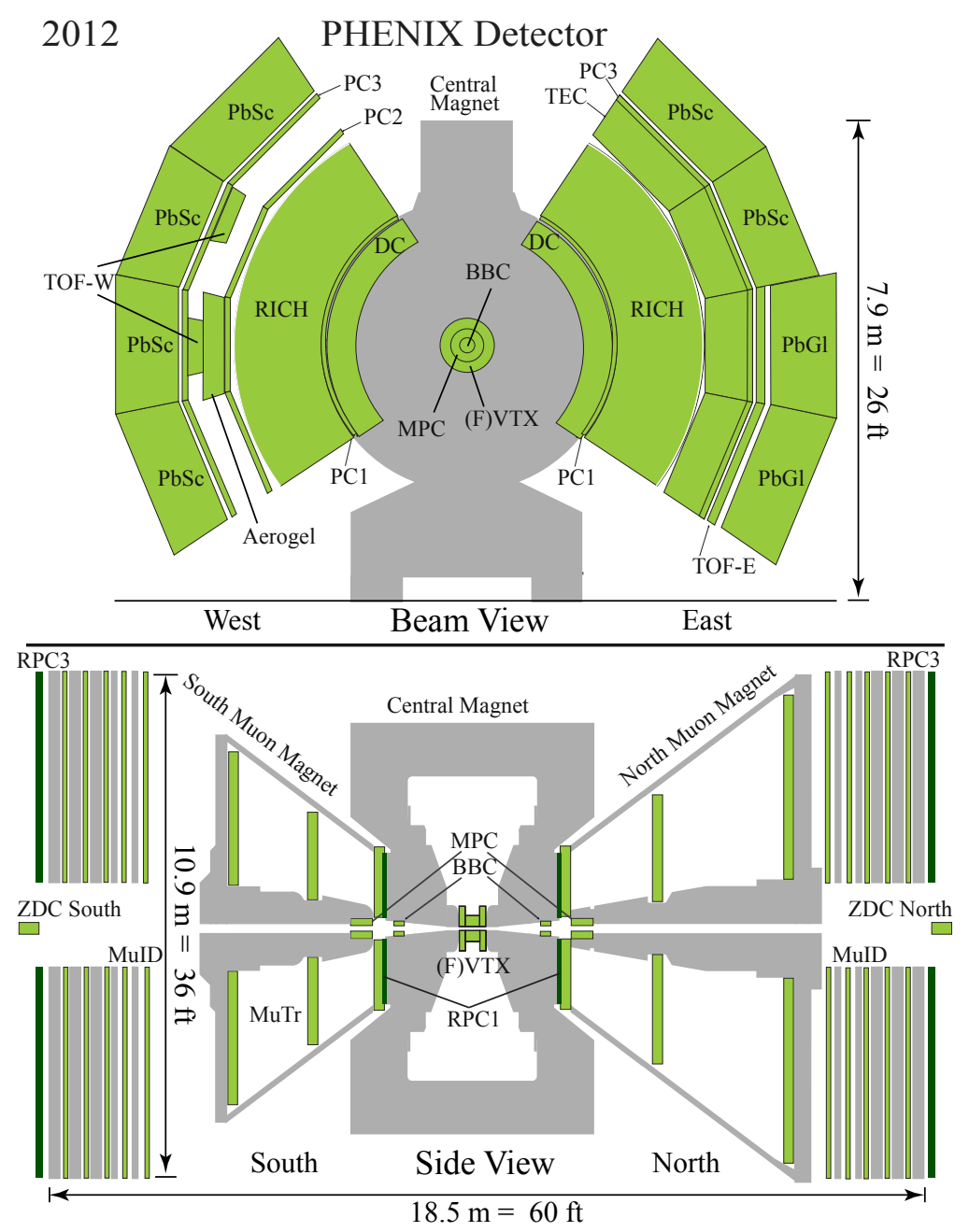

Figure 1: Overview of the PHENIX apparatus; upper panel shows the central arms, while the lower panel shows the muon arms, the Beam-Beam Counters (BBCs), and the Zero Degree Calorimeters (ZDCs).

\section{The PHENIX Experimental Apparatus}

PHENIX operated as a collider experiment at the Relativistic Heavy-Ion Collider (RHIC) at Brookhaven National Laboratory (BNL) from 2001 to 2016. It comprised two major detector systems:

- a pair of central arms covering the rapidity range $|y|<0.35$ including vertex detectors, a bending magnet, tracking detectors, particle identification and calorimetry (upper panel of Figure 1)

- a pair of muon arms covering the forward and backward rapidity range $1.4<|y|<2.4$ including vertex detectors, muon range shielding, muon tracking, a radial magnetic field, and a muon/hadron identifier (lower panel of Figure 1) 
In addition, at very forward rapidity a pair of Zero Degree Calorimeters (ZDCs), located beyond the accelerator bending magnets (see the lower panel of Figure 1), detected neutrons emitted from the collisions.

The experimental data comes from the 2015 running period, a unique situation in which a transversely polarized proton beam collided with either another beam of transversely polarized protons or a beam of unpolarized $\mathrm{Al}$ or Au nuclei, with a nucleon-nucleon center-of-mass energy of $\sqrt{s_{N N}}=200 \mathrm{GeV}$.

\section{TSSA for $\pi^{0}$ Production at Central Rapidity}

This study used the tracking and calorimetric abilities of the central arms to reconstruct $\pi^{0}$ particles from pairs of electromagnetic showers not associated with any charged particle in the trackers. The TSSA for $\pi^{0}$ production in $\vec{p}+p, \vec{p}+\mathrm{Al}$, and $\vec{p}+\mathrm{Au}$ collisions is consistent with zero over the transverse momentum range $1<p_{T}<16 \mathrm{GeV}$. Integrating over that $p_{T}$ range, we display the A-dependence of the asymmetry in Figure 2, and the lack of any A-dependence at central rapidity is very clear. A manuscript describing this measurement is in preparation.

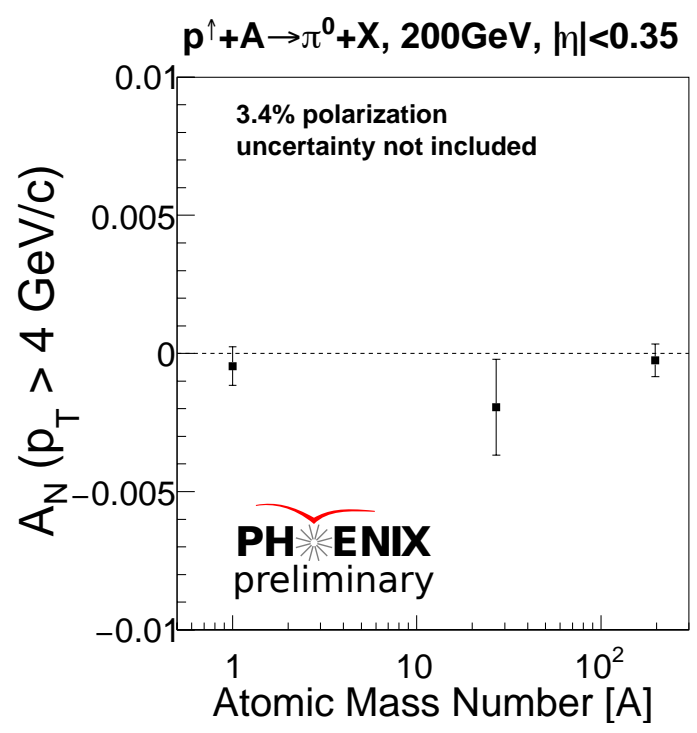

Figure 2: The A-dependence of the transverse single spin asymmetry in $\pi^{0}$ production.

\section{TSSA for $J / \psi$ Production at Forward and Backward Rapidity}

The TSSA for $J / \psi$ production in $\vec{p}+p$ collisions at forward and backward rapidity was previously measured in PHENIX and was seen to be consistent with zero; in the 2015 run we were able to observe if this asymmetry had any nuclear dependence. We observe a negative asymmetry in $\vec{p}+\mathrm{Au}$ collisions in both forward and backward production; the largest signal is seen for $-0.11<x_{F}<-0.05$, as is seen in Figure 3. Looking at the results instead as a function of $p_{T}$, 
Figure 4 , we observe a negative asymmetry in $\vec{p}+$ Au collisions in both forward and backward production for $0.42<p_{T}<2 \mathrm{GeV}$. This work was published in [3].

It is evident that the nuclear environment has created or enhanced the asymmetry. This could be a new avenue for insight into the $J / \psi$ production mechanism in nuclei, critical for an understanding of $J / \psi$ production modification in $A+A$ collisions.

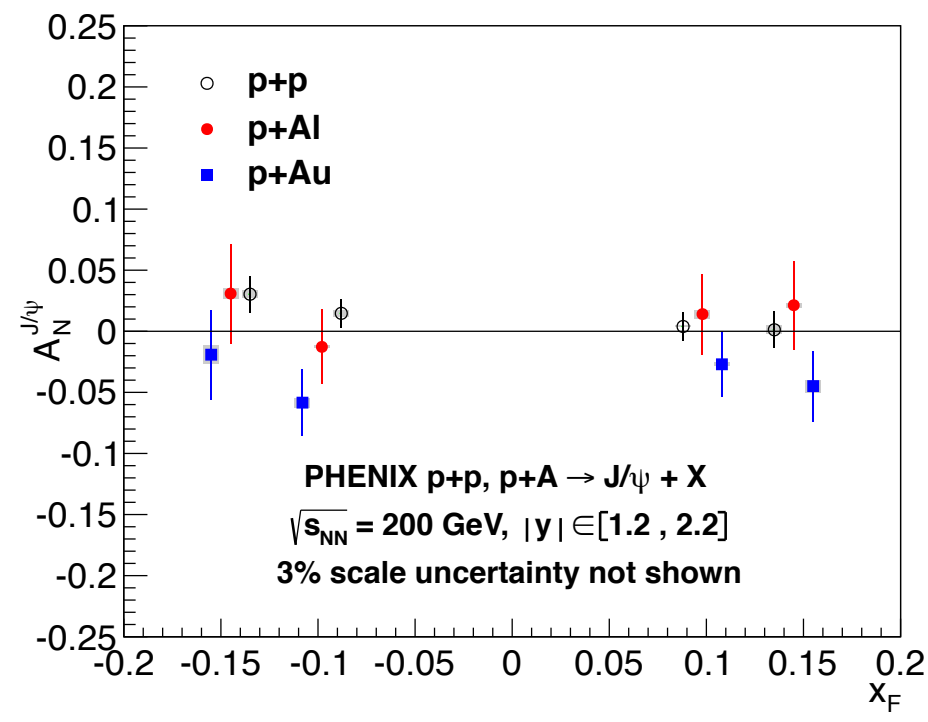

Figure 3: The nuclear dependence of the TSSA in $J / \psi$ production, as a function of $x_{F}$.
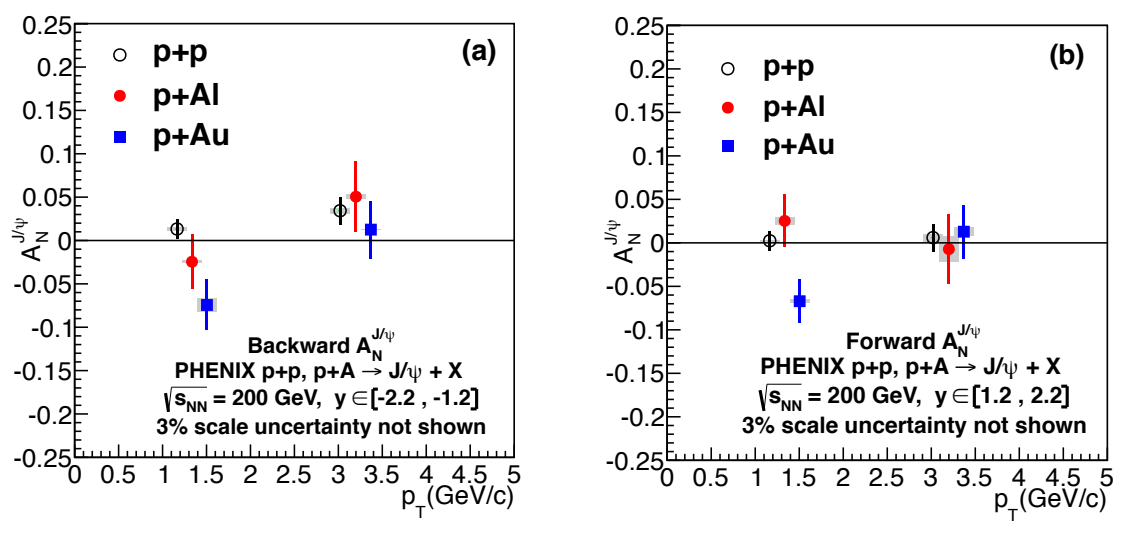

Figure 4: The nuclear dependence of the TSSA in $J / \psi$ production, as a function of $p_{T}$.

\section{TSSA for $\pi^{+} / K^{+}$Production at Forward Rapidity}

The asymmetry in the production of charged pions at forward rapidity, first seen in experiments at Argonne National Laboratory [11] and also seen much more recently at RHIC by BRAHMS [6], was the asymmetry that started the investigation of TSSAs 40 years ago. With the 2015 data at 
RHIC we had the opportunity to study the nuclear dependence of this very fundamental production channel. This study in PHENIX used the same muon arms as the study of $J / \psi$ production described above, but used the Muon Identifier arms to exclude muon tracks, thus providing a sample of unidentified hadron tracks composed almost entirely of pions and kaons.

In Figure 5 are shown sinusoidal fits to the azimuthal distribution of the spin asymmetry. A very strong suppression of the azimuthal asymmetry is seen for increasing nuclear mass number. In Figure 6 we show a fit of the asymmetries to a function of the form $\mathrm{A}^{-\alpha / 3}$ so as to quantitatively describe the nuclear dependence. The best fit is for $\alpha=1.21_{-0.52}^{+1.08}$. This is consistent with the expectation of Hatta et al. [9] that there should be an $\mathrm{A}^{1 / 3}$ suppression of the final-state Collins asymmetry due to gluon saturation. Note that the possibility of "no mass dependence" $(\alpha=0)$ is ruled out. Finally in Figure 7 we display a fit of the asymmetry, binned into centrality classes, as a function of the average number of binary collisions $N_{\text {coll }}^{\text {Avg }}$ calculated for each centrality class range. The fit is to a function of the form $\left(N_{\text {coll }}^{\text {Avg }}\right)^{-\beta}$, and the best fit gives $\beta=1.19_{-0.47}^{+0.74}$. Once again, the possibility of "no mass dependence" ( $\beta=0$ in this case) is ruled out. A manuscript describing this measurement is in preparation.

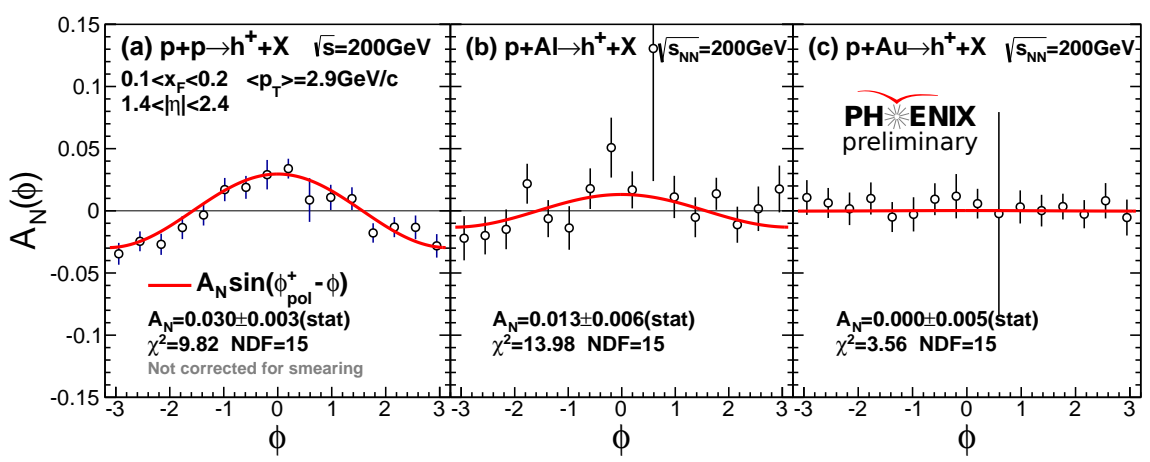

Figure 5: The nuclear dependence of the TSSA in $\pi^{+} / K^{+}$production. Each panel displays a sinusoidal fit of the azimuthal asymmetry of the yield, for $\vec{p}+p, \vec{p}+\mathrm{Al}$, and $\vec{p}+\mathrm{Au}$ interactions.

\section{TSSA for Neutron Production at Very Forward Rapidity}

The discovery at RHIC some years ago of the large TSSA for very forward neutron production in $\vec{p}+p$ collisions $[7,1]$ was already a surprising result; the RHIC 2015 data presented an opportunity to see if this asymmetry had a nuclear dependence. The principal detector in use for this study was the ZDC (zero-degree calorimeters) mentioned earlier; they are positioned beyond the bending magnets used to steer the beams into collision and so are sensitive primarily to neutrons arising from the interaction point. In addition, the BBC (beam-beam counters) were used with the ZDC to create three different trigger conditions, to study the centrality dependence.

1. ZDC Inclusive: ZDC energy $>15 \mathrm{GeV}$ (all collisions with significant ZDC energy)

2. ZDC $\otimes$ BBC-tag: ZDC Inclusive AND $\geq 1$ hit in each BBC (hard/central collisions)

3. ZDC $\otimes \mathbf{B B C}$-veto: ZDC Inclusive AND no hit in any BBC (diffractive/peripheral collisions) 

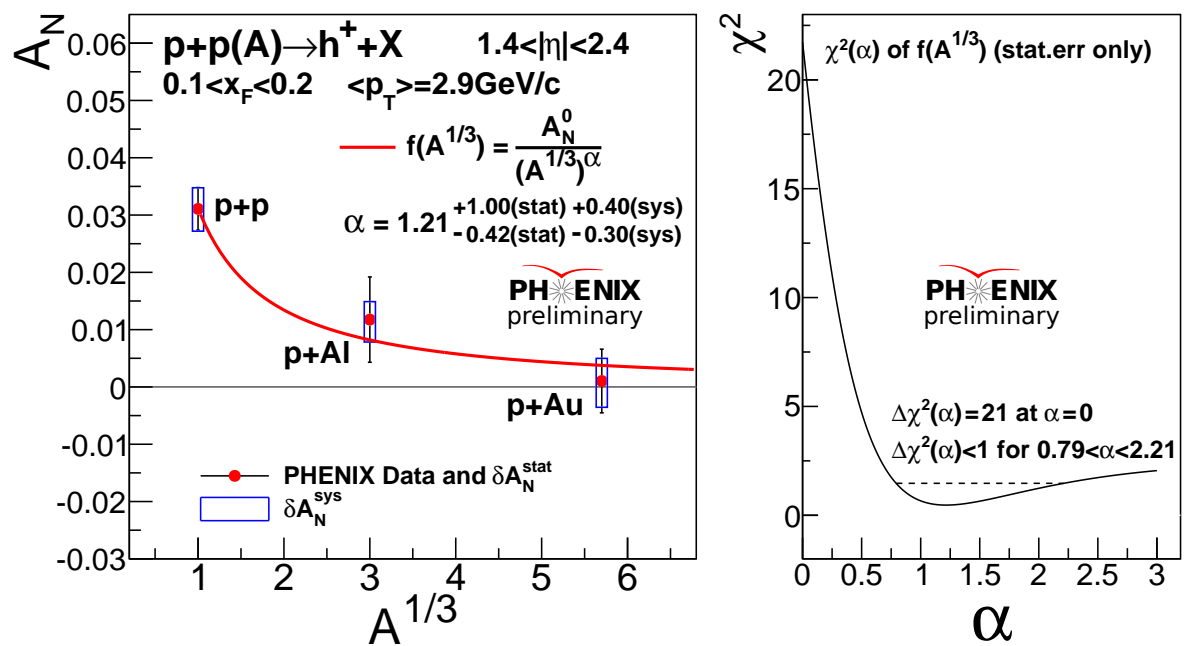

Figure 6: Demonstration of the $\mathrm{A}^{1 / 3}$-suppression of the TSSA in $\pi^{+} / K^{+}$production.
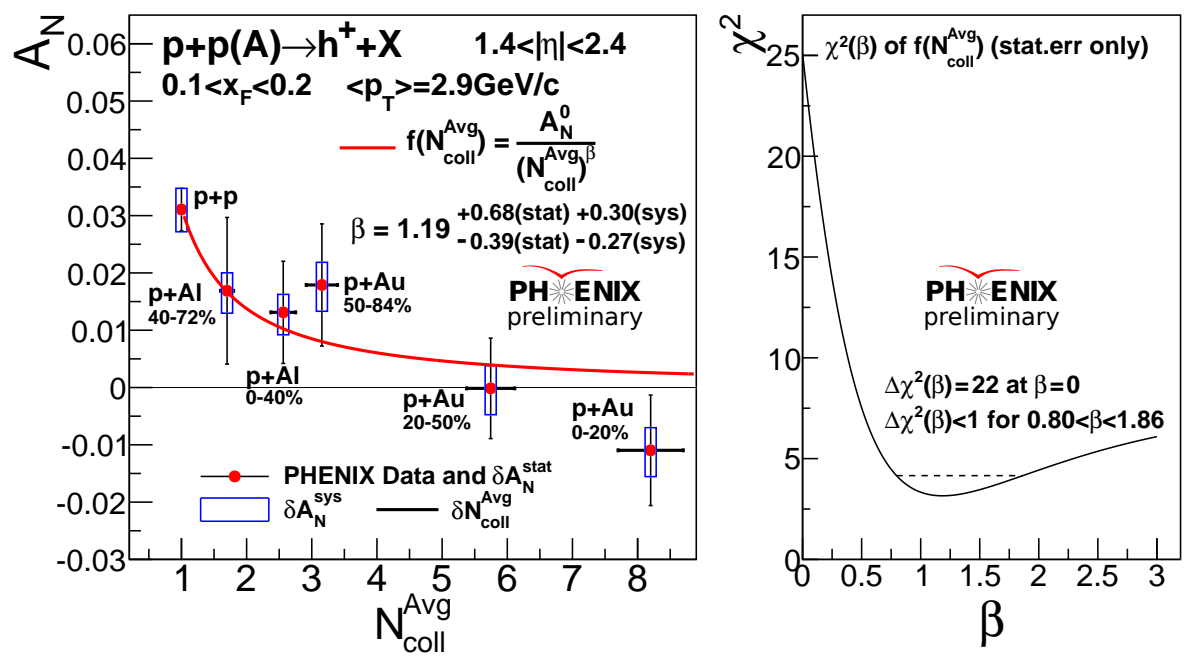

Figure 7: Demonstration of the $N_{\text {coll }}^{\mathrm{Avg}}$-suppression of the TSSA in $\pi^{+} / K^{+}$production. The $p+\mathrm{Al}$ and $p+\mathrm{Au}$ data points are labeled with the associated centrality class, given as a range of percentages; the most central collisions have the lowest centrality percentage.

The results for each of these triggers are shown in Figure 8. Collisions with small impact parameter (BBC-tag) show a negative asymmetry with a moderate nuclear dependence. On the other hand, collisions with a large impact parameter (BBC-veto) show a strong nuclear dependence, indicating the importance of ultra-periphercal collisions. Mitsuka [12] has shown that in an ultra-peripheral collision the photon flux from the unpolarized nucleus can interact with the polarized proton to produce such asymmetries. These measurements have been published in [2]. 


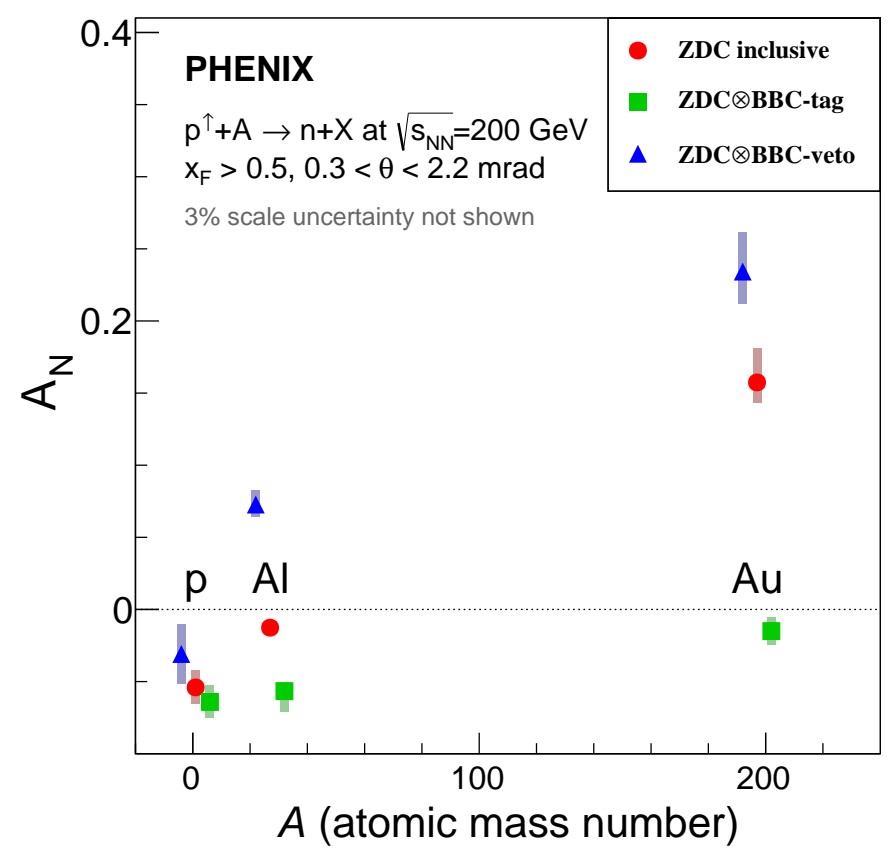

Figure 8: Nuclear- and centrality-dependence of the TSSA in neutron production at very forward rapidity.

\section{Summary of Nuclear Dependence of TSSAs}

The RHIC 2015 data with $\vec{p}+p, \vec{p}+\mathrm{Al}$, and $\vec{p}+\mathrm{Au}$ collisions has been used by PHENIX to study the transverse single spin asymmetries in a number of systems.

- $\pi^{0}$ production at central rapidity: the TSSA is very small, independent of nuclear size

- $J / \psi$ production at forward and backward rapidity: the TSSA is very small in $\vec{p}+p$, becoming negative at low $p_{T}$ in $\vec{p}+\mathrm{Au}$

- $\pi^{+} / K^{+}$production at forward rapidity: the TSSA is very large in $\vec{p}+p$, and is then strongly suppressed with increasing nuclear size and centrality, suggesting a gluon saturation effect

- neutron production at very forward rapidity: largest effect seen in ultra-peripheral collisions

A rich variety of behavior is revealed in this cross between spin-dependent scattering phenomena and the physics of cold nuclear matter.

\section{Acknowledgements}

This work was supported by a grant from the US Department of Energy, Office of Science, Medium Energy Nuclear Physics program. 


\section{References}

[1] A. ADARE ET AL., Inclusive cross section and single transverse spin asymmetry for very forward neutron production in polarized $p+p$ collisions at $\sqrt{s}=200 \mathrm{GeV}$, Phys. Rev. D, 88 (2013), p. 032006.

[2] C. AidAla ET AL., Nuclear dependence of the transverse-single-spin asymmetry for forward neutron production in polarized $p+$ a collisions at $\sqrt{s_{N N}}=200 \mathrm{GeV}$, Phys. Rev. Lett., 120 (2018), p. 022001.

[3] _ - Single-spin asymmetry of $\mathrm{J} / \psi$ production in $p+p, p+\mathrm{Al}$, and $p+\mathrm{Au}$ collisions with transversely polarized proton beams at $\sqrt{s_{N N}}=200 \mathrm{GeV}$, Phys. Rev. D, 98 (2018), p. 012006.

[4] M. Anselmino, M. Boglione, U. D’Alesio, E. Leader, S. Melis, F. Murgia, And A. PROKUDIN, Role of Collins effect in the single spin asymmetry $A_{N}$ in $p^{\uparrow} p \rightarrow h X$ processes, Phys. Rev. D, 86 (2012), p. 074032.

[5] M. Anselmino, M. Boglione, U. D’Alesio, S. Melis, F. Murgia, And A. Prokudin, Simultaneous extraction of transversity and Collins functions from new semi-inclusive deep inelastic scattering and $e^{+} e^{-}$data, Phys. Rev. D, 87 (2013), p. 094019.

[6] I. ARSENE ET AL., Single-transverse-spin asymmetries of identified charged hadrons in polarized pp collisions at $\sqrt{s}=62.4 \mathrm{GeV}$, Phys. Rev. Lett., 101 (2008), p. 042001.

[7] A. BAZILEVSKY ET AL., Measurement of single transverse-spin asymmetry in forward production of photons and neutrons in p p collisions at $\sqrt{s_{N N}}=200 \mathrm{GeV}$, AIP Conf. Proc., 675 (2003), pp. 584-588.

[8] J. Collins, Fragmentation of transversely polarized quarks probed in transverse momentum distributions, Nuclear Physics B, 396 (1993), pp. 161 - 182.

[9] Y. Hatta, B.-W. XiaO, S. Yoshida, And F. YUAn, Single spin asymmetry in forward pA collisions. II. Fragmentation contribution, Phys. Rev. D, 95 (2017), p. 014008.

[10] K. Kanazawa, Y. Koike, A. Metz, And D. Pitonyak, Towards an explanation of transverse single-spin asymmetries in proton-proton collisions: The role of fragmentation in collinear factorization, Phys. Rev. D, 89 (2014), p. 111501.

[11] R. D. Klem, J. E. Bowers, H. W. Courant, H. Kagan, M. L. Marshak, E. A. Peterson, K. RudDick, W. H. Dragoset, AND J. B. Roberts, Measurement of asymmetries of inclusive pion production in proton-proton interactions at 6 and $11.8 \mathrm{GeV} / c$, Phys. Rev. Lett., 36 (1976), pp. 929-931.

[12] G. MitsuKA, Recently measured large $A_{N}$ for forward neutrons in $p^{\uparrow} A$ collisions at $\sqrt{s_{N N}}=200$ GeV explained through simulations of ultraperipheral collisions and hadronic interactions, Phys. Rev. C, 95 (2017), p. 044908.

[13] D. Pitonyak, Transverse spin observables in hard-scattering hadronic processes within collinear factorization, Int. J. Mod. Phys., A31 (2016), p. 1630049.

[14] D. SIVERS, Single-spin production asymmetries from the hard scattering of pointlike constituents, Phys. Rev. D, 41 (1990), pp. 83-90. 